group (CG, $\mathrm{n}=52$ ). Tirofiban was only administrated in the tirofiban group. Before the CAG, enough clopidogrel, aspirin and heparin be used in both groups. The MACE and the haemorrhage events were collected in each group during in-hospital. The lesion and reperfusion of the IRA and myocardial were analyses by QCA and TMPG. The platelet aggregation rate were recorded All patients received UCG 1 week and 24 weeks after PCI to evaluate the heart function. Results There was no significant differences in age, gender, risk factors, pre-angina, the location of the AMI, heart function, and the mean interval from onset to PCI between the two groups. A greater percentage of TIMI 1 flow of IRA was achieved in TG compared with the control group before PCI $(p<0.05)$. The percentage of TIMI 3 flow of IRA after the guild wire first crossing was higher $(p<0.05)$ in TG. The percentage of TIMI 3 flow in TG after PCI was higher than that in CG $(p<0.05)$. The CTFC and slow-reflow phenomenon was fewer $(p<0.05)$ in TG after PCI. The percentage of TMPG beyond 2 grade was higher in TG $p<0.05)$. The value of LVEF 1 week after PCI in TG was higher than that in CG $(p<0.01)$. The platelet aggregation rate in TG was lower after tirofiban administration for $0.25,0.5,2,6$ and $12 \mathrm{~h}$. There was no significant difference in haemorrhage events between the two groups. There was a lower incidence of MACE in TG compared with that in CG during in-hospital and follow up.

Conclusion Intravenous administration of trofiban can inhibit the platelet aggregation, improve the coronary flow of IRA, decrease the incidence of NRP in AMI patients performed PCI, which in turn will improve the heart function and decrease the incidence of MACE. Tirofiban can make more IRA patent before PCI, but do not increase the haemorrhage events.

\section{e0656 THE EFFECT OF RECOMBINANT HUMAN B-TYPE NATRIURETIC PEPTID ON CORONARY CIRCULATION AND RENAL HAEMODYNAMICS IN YORK PIGS MODEL OF ACUTE MYOCARDIAL INFARCTION WITH HEART FAILURE}

doi:10.1136/hrt.2010.208967.656

Fu Xianghua, Zhang Jing, Wang Xuechao, Wang Yanbo, Xue Ling, Fan Weize, Wu Weili, Jiang Yunfa. The 2nd Hospital of Hebei Medical University, Shijiazhuang, Hebei, China

Objective To evaluate the impact of intravenous administration of rhBNP on coronary and renal artery haemodynamics in York pigs model of AMI-ADHF.

Methods 14 York pigs were included in this study. After the AMI$\mathrm{ADHF}$ models were established, pigs were randmized into saline group and rhBNP group. Coronary pressure $\left(\mathrm{P}_{\mathrm{c}}\right)$, the average peak velocity (APV), coronary vascular resistance (CR), coronary flow reserve (CFR) and coronary diameter were recorded simultaneously at baseline, instant after the model established, 60 min after continuous infusion of $0.01 \mu \mathrm{g} \cdot \mathrm{kg}^{-1} \cdot \mathrm{min}^{-1} \mathrm{rhBNP}$ and the time point of LVEDP $<12 \mathrm{~mm} \mathrm{Hg}$. The blood flow of the coronary were measured at rest and maximal hyperaemia. Renal angiography was performed by $4 \mathrm{~F}$ catheter and quantitative measurement of diameter was recorded by the computer assisting system. The average peak rate of renal artery $\left(\mathrm{APV}_{\mathrm{ra}}\right)$ was recorded, determination of quantitative angiography of renal artery diameter, renal vascular resistance. LVEDP and LVEF was measured.

Results 1. Coronary artery diameter increased after rhBNP administration. APV and CBF were significantly increased and CR decreased after rhBNP administration. CFR was significant rebound after continuous infusion of $0.01 \mu \mathrm{g} \cdot \mathrm{kg}^{-1} \cdot \mathrm{min}^{-1} \mathrm{rhBNP}$ for $30 \mathrm{~min}$. APV and CBF significantly increased and CR significantly decreased at the stage of infusion $0.010 \mu \mathrm{g} \cdot \mathrm{kg}^{-1} \cdot \mathrm{min}^{-1} \mathrm{rhBNP}$ in $\mathrm{rhBNP}$ Group. 2. Renal artery pressure was significantly lower after rhBNP administration. RhBNP exerts renal vasodilator effects in a dose related relationship. RBF increased gradually after administration of rhBNP and was significantly higher than control group. RVR decreased after administration of rhBNP. LVEF was lower than baseline after the models established and tended to increase after administration of rhBNP.

Conclusion It could increase blood flow of injury coronary artery, improve CFR and improve the coronary and renal haemodynamics after intravenous administration of rhBNP in pigs with AMI-ADHF.

\section{e0657 THE PERIOPERATION EFFECTS OF RECOMBINANT HUMAN B-TYPE NATRIURETIC PEPTIDE FOR HEART FAILURE PATIENTS WITH PRIMARY PERCUTANEOUS CORONARY INTERVENTION}

doi:10.1136/hrt.2010.208967.657

Fu Xianghua, Zhang Jing, Wang Xuechao, Wang Yanbo, Hao Guozhen, Fan Weize, Jiang Yunfa. The 2nd Hospital of Hebei Medical University, Shijiazhuang, Hebei, China

Objective To study the efficacy and safety of recombinant human Btype natriuretic peptide (rhBNP) in AMI-ADHF patients undergoing $\mathrm{PCI}$, especially changes in renal function and the impact of shortterm outcome during BNP treatment.

Methods 87 consecutive patients with AMI-ADHF entrolled in the study. All patients were randomly assigned to the rhBNP group and control group. rhBNP was given at $1.5 \mu \mathrm{g} \cdot \mathrm{kg}^{-1}$ intravenously and then infused intravenously $\left(0.0075-0.030 \mu \mathrm{g} \cdot \mathrm{kg}^{-1} \cdot \mathrm{min}^{-1}\right) .0 .9 \%$ Saline was used intravenously in control group as control. Clinical symptoms and killip grade were recorded. Plasma BNP levels were meaured before and after stopping the drug $6 \mathrm{~h}, 14$ days, 30 days. LVEDD and LVEF was measured. Serum creatinine (Scr) was measured before and after administered the medication $24 \mathrm{~h}, 48 \mathrm{~h}$, $72 \mathrm{~h}, 7$ days and 14 days using simplified MDRD equation to calculate estimated glomerular filtration rate. Recording the major adverse cardiac events occurrence within 30 days.

Results rhBNP group has a less dyspnoea time than the control group; The plasma BNP levels significantly lower than before treatment at different time point in the two groups. The LVEF was significantly higher in treatment group compared with baseline levels after treatment $24 \mathrm{~h}$, while LVEDD significantly decreased even after discontinuation the treatments, which remain so when the 30 days. The LVEF and LVEDD improvements in rhBNP group were significantly better than in the control group after treatment 24 h, 14 days. At day 7 after PCI, the SCr had lowered to the baseline level in the rhBNP group. The estimated glomerular filtration rate after PCI was higher in the rhBNP group than that in the control group. The occurrence of CIN was significantly lower in the rhBNP group than in the control group. The MACE event of $30 \mathrm{~d}$ in rhBNP group was significantly lower than the control group.

Conclusion rhBNP can promptly and effectively improve the heart function, reduce the incidence of major adverse cardiac events rate in acute myocardial infarction with heart failure patients, which also had a renal function protective effect in patients with and decreased incidence on CIN.

\section{e0658 ESTABLISHMENT OF YORK PIG MODEL OF ACUTE MYOCARDIAL INFARCTION WITH ACUTE DECOMPENSATED HEART FAILURE BY CORONARY OCCLUSION WITH BALLOON AND INJECTING OF MICROEMBOLUS}

doi:10.1136/hrt.2010.208967.658

Fu Xianghua, Zhang Jing, Wang Xuechao, Wang Yanbo, Miao Oing, Jiang Yunfa, Hao Guozhen, Gu Xinshun. The 2nd Hospital of Hebei Medical University, Shijiazhuang, Hebei, China

Objective To evaluate the method of yorkpig model of AMI-ADHF by coronary occlusion with balloon and injecting of microembolus 
and evaluate the change of coronary and renal haemodynamic parameters on York pig model on AMI-ADHF.

Methods 10 York pigs were selected in this trial. LAD was occluded by balloon which was placed on mid-distal for 30-45 min until ST segment elevation for $15 \mathrm{~min}$. After deflating the balloon, the sterile microembolus were injected into LAD intermittently and injecting stopped until LVEDP $>18 \mathrm{~mm} \mathrm{Hg}$. The coronary pressure and flow velocity were recorded simultaneously at baseline, instant, $30 \mathrm{~min}$ and 60 min after AMI-ADHF model success. Average peak velocity (APV), coronary resistance and coronary flow reserve were obtained at basic and maximal hyperaemia. Renal artery angiography was performed and renal artery diameter and pressure was measured. Doppler flowire was placed in renal artery for measure of APV of renal artery $\left(\mathrm{APV}_{\mathrm{ra}}\right)$, renal artery vascular resistance and renal blood flow. At the same time ECG were recorded per $15 \mathrm{~min}$. CK-MB and TnI were measured in all York pigs at baseline, $2 \mathrm{~h}, 4 \mathrm{~h}, 8 \mathrm{~h}, 12 \mathrm{~h}$ and $24 \mathrm{~h}$ after reperfusion. Cardiac ultrasonograph was performed to evaluate the heart function.

Results (1) According to the standards of AMI-ADHF, eight models were successfully established. (2) Basic and hyperaemia APV were all decreased significantly, they were lower than that of before AMIADHF. coronary resistance was increased after AMI-ADHF model success. coronary flow reserve was lower than that of baseline. (3) Renal artery pressure has increased trend than baseline. renal artery vascular resistance was increased, APVr and renal blood flow was decreased than baseline.

Conclusion A stable experimental method of York pig model of AMIADHF was established successfully by coronary occlusion with balloon and injecting of microembolus in LAD. The method had advantages of closed chest, higher succeed rate and stablility to those of drug induced, tachycardia-pacing induced, coronary artery ligation induced or microsphere injection alone.

\section{e0659 THE EFFECT AND SAFETY OF TIROFIBAN COMBINED CLOPIDOGREL IN AMI PATIENTS WITH PCI VIA TRANSRADIAL APPROACH}

doi:10.1136/hrt.2010.208967.659

Fu Xianghua, Hao Oingqing, Fan Weize, Zhang Jing, Gu Xinshun, Jiang Yunfa, Hao Guozhen. The 2nd Hospital of Hebei Medical University, Shijiazhuang, Hebei, China

Objective To evaluate the effect and safety of clopidogrel plus tirofiban in percutaneous coronary intervention (PCI) of acute myocardial infarction via transradial approach.

Methods A total of 104 patients with ST segment elevation myocardial infarction who were underwent emergency PCI were randomised into two groups: clopidogrel combined tirofiban group $(n=48)$ and clopidogrel group $(n=56)$. Bolus tirofiban $(10 \mu \mathrm{g} / \mathrm{kg})$ was infused in $3 \mathrm{~min}$ before PCI and then $0.15 \mu \mathrm{g} / \mathrm{kg} / \mathrm{min}$ continued pumping for $24 \mathrm{~h}$ in the clopidogrel combined tirofiban group, while saline was used in the same way in the clopidogrel group. The TIMI flow grade in IRA before PCI, after the first guide wire getting through and instant post-PCI were recorded and compared between the two groups. The myocardial blush grade post-PCI and heart function were analysed and compared between the two groups. Hemorrhagic complications and MACE in-hospital were monitored and followed up.

Results The percentage of TIMI flow grade 1 of IRA before PCI was higher in the clopidogrel combined tirofiban group than that in the clopidogrel group $(p<0.05)$. The percentage of TIMI flow grade 3 of IRA after the guide wire first crossing through the occlusion segment was higher in clopidogrel combined tirofiban group than that in the clopidogrel group $(\mathrm{p}<0.05)$. The percentage of TIMI flow grade 3 of IRA after PCI was also higher in clopidogrel combined tirofiban group than that in the clopidogrel group $(p<0.05)$. The corrected TIMI flow count (cTFC) in the clopidogrel combined tirofiban group was smaller to the clopidogrel group $(p<0.05)$. LVEF at 1 week after PCI in the tirofiban group was higher than that in the clopidogrel group $(p<0.01)$. There was no significant difference in hemorrhagic complications between the two groups. The incidence rate of MACE during in-hospital in the clopidogrel combined tirofiban group was lower compared to that in the clopidogrel group $(\mathrm{p}<0.05)$.

Conclusions Clopidogrel combined tirofiban used during the emergency PCI for AMI via transradial approach can quickly inhibit the platelet aggregation, decrease thrombosis event, improve blood flow of IRA and increase the perfusion of myocardial microcirculation as well preserve the function of left ventricle and reduce the incidence rate of MACE during in-hospital, while not associate with increasing the incidence rate of serious haemorrhage complications.

\section{e0660 OUALITY OF LIFE AFTER FOUR TIMES INTRAVENOUS INFUSION OF BONE MARROW MSCS FOR HEART FAILURE PATIENTS}

doi:10.1136/hrt.2010.208967.660

${ }^{1}$ Oi Chunmei, ${ }^{2} \mathrm{Hao} \mathrm{Ji},{ }^{2}$ Zhang Xueshan, ${ }^{2}$ Feng Jianqi, ${ }^{2}$ Diao Jun, ${ }^{2}$ Wu Weiheng. ${ }^{1}$ Department of Cardiology, The Second Hospital Affiliated to Xuzhou Medical College; ${ }^{2}$ Department of Cardiology, The Second Hospital Affiliated to Xuzhou Medical College

Objective Heart failure is a complex clinical syndrome that can result from any structural or functional heart disorder, so the patients have marked limitation of physical activity and poor life quality. Some experiments have tested the safety and efficacy of intravenously injection of MSCs to restore the heart function, the goal of this study is to verify patients will have a better quality of life after stem cell transplantation.

Methods The study involved 85 acute myocardial infarction patients with heart failure who had ejection fractions less than $45 \%$. Between May 2008 and January 2010, 43 consecutive heart failure patients underwent MSCs injcetion through vein for four times in the experimental group. The number of cells prior to injection was 41.6 \pm 34.7 million cells, another 42 heart failure patients were as control group. NYHA class, 6 min walk test, Brain natridiureticpeptide level, transthoracic echocardiography, single-photon emission CT are obtained prior to transplatation, the patients who did not received PCI examed by MRI. Quality of life evaluated by SF36. These tests are repeated at 6 months.

Results At six-month follow-up, transplantation of MSCs enhanced left-ventricular systolic function $(39.12 \pm 4.54) \%$ versus $(52.08 \pm 1.02) \%$, emission CT $(40.19 \pm 3.18) \%$ versus $(53.18 \pm 2.15) \%$, BNP declined $(429 \pm 39) \mathrm{pg} \cdot \mathrm{ml}^{-1}$ versus $(152 \pm 19) \mathrm{pg} \cdot \mathrm{ml}^{-1}$, quality of life of the stem cell transpaltation patients were significantly improved according to the assessment of physical functioning, role limitations due to physical health, bodily pain, general health perceptions and vitality, but the social functioning, role limitations due to emotional problems and mental health were not improved. NYHA class decreased $(2.08 \pm 0.25)$ versus $(1.85 \pm 0.38), 6 \mathrm{~min}$ walk test increased $(328.3 \pm 45.3)$ versus $(356.8 \pm 43.2)$. MRI examinantion also observed the decreased infarct size in the experimental group.

Conclusion Quality of life and ejection fraction were significantly improved after Bone marrow MSCs injected through four times intravenous infusion. 